\title{
Un Documento sobre la Rebelión del Cacique Túpac Amaru.
}

\section{INTRODUCCION}

Entre los manuscritos salvados del incendio de la antigua Biblioteca Nacional se encuentra uno, bajo el rubro de "Literatura Incásica. Justo Apu Saluuaraurá. 1838". Lí noticia bibliográfica se debe a Ella Dumbar Temple, en el "Boletín de la Biblioteca Nacional", año I, n" I, X-94.3, pl). I4-I $5, n^{2}$ I 5 .

El manuscrito contiene 8 partes, que presentan un contenido distinto. En la ocasión presente interesa la colocada en sexto lugar, y cuya denominación es: "Noticia importante para el conocimiento de la posteridad". De acuerdo con la Sumilla, además de las noticias sobre la rebelión del cacique Túpac Amaru, se añaden la Sentencia de Areche contra el jefe rebelde y la Carta de éste al Visitador. La Sentencia se encuentra en el tomo V de la Colección de Pedro Angelis ( $r . "$ edición 1836) y también en los Documentos Históricos de M. de Odriozola, t. I, quien, en lo referente a Túpac Amaru, copia a Angelis en su casi totalidad. En cuanto a la Carta (que dirigió el Cacique rebelde al Visitador, desde Tinta, el 5 de narzo de I78I), no la trae Angelis, pero si Odriozola en la colección y tomo mencionados, ocupando desde la página I I4 hasta la 
página 153 Sahuaraura dice, claramente, de donde tomó la carta $y$ la sentencia mencionadas, estampando en una nota correspondiente a la página 2 I 3 : "son sacados de las piesas (sic) oficiales que el Virrey Marques de Abiles mandó recoger".

Respecto al manuscrito, existe un error en su numeración. Pues de la página 180 se pasa a la 182 , mientras que el texto no acusa vacio alguno en la continuidad de su narración.

Finalmente, sobre la personalidad del autor, don Justo Apu Sahuaraura, remitimos a su propia obra: "Recuerdo de la monarquía indiana" (donde existe un retrato de Sahuaratura). Su genealogía ha sido estudiada por Ella Dumbar Temple en "La descendencia de Huayna Capac" (Rev. Histórica $1937-40$ ) y con mayor detenmiento en su tesis doctoral "La estirpe de Huayna Capac" (Lima I946), lo que permite señalar, de manera crítica, su entronque imperial y su calidad de legítimo descendiente de Paullu Inca, hermano del famoso inca Manco II. A continuación se inserta la "Noticia Importante para el conocimiento de la posteridad" de Justo Apu Sahtiaraura.

\section{NOTICIA IMPORTANTE PARA EL CONOCIMIENTO DE LA POSTERIDAD}

"EI Bravo y virtuoso Don Jose Grabiel Tupac Amaru (1), fúé educado en el Colegio de San Franciseo de Borja (2) de esta Cin-

(1) Los apellidos propios del Caulillo eran Condorcanqui (paterno) y Noguera (materno). El apellido genérico Túpac Amaru le venía por ser descendiente directo por línea femenina del íltimo inea Túpne Amaru.-Véso "Doeumentos sobre la rebelión del cacique Túpac Amaru, por Carlos Valefreel. Bol. Bibliográfico de la U. de San Marcos, nos. 3-4, 1944, pp. 202, grupo A, doc. N.o 1.-."Genealogía de Túpac Amaru", pub. Francisco A. Loayza, serie $I$, tomo $X$. Imp. Miranda, Lima 1946.

(2) Colegio de Caciques creado durante el gobierno del virrey Príncipa de Esquilache, pero cuyos antecedentes se remontan a los tiempos del virrey don Franciseo de Toledo. 
dad del Cuzeo, $y$ poseia rirtudes gue en la vida privata, liv harian un hombre rligno de ser querido, y lespetarlo; pero parere iat de aljure.

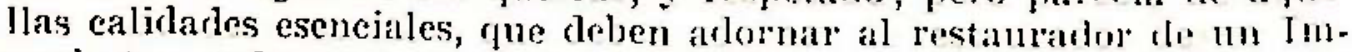
perio tan poderoso como el Perú. (9) Su ayre era nolles sus mantras impouentes, de estatuma regular, y trazil robusta, anmoso, $y$ emprendedor, pere de pationes vinlentas y de eomocintentes, $y$ miras

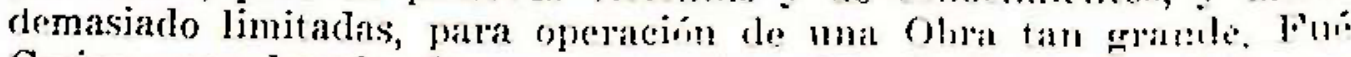
Casique por derecho de sangre en el pueblo de 'l'ungitile:al (.t) en la Provincia de Tinta, y era de la descendencia del Prineipo Don Felipe Tupac Amaro (5), a quien ol Virrey Ton Franciseo Tolede, con engaños le hizo salcar de las Montañas de Villeapampa, el año de 1562, y le hizo cortar la cabesa, en la plaza grande do estia Cindial: tonde se habia refugiedo, $y$ formado una pergucna Monarquia su Padre el Inca Nanco, por hallarse arrelataclo t!el Imperio do sus Pardres, por los Pizarros, Almarros y Compañons. El Comiciomilato para la pricion del dicho Dom Felipe T'upas: Amaro, fuí Don Mastin de Loyolia (6); sobrino de Sin Trnacio de: Troyola.

"Dicho Don Jose Grabiel T'upac Amaro, eorno descendicute de los soberanos Incas quiso reasumir al TuTerio red Peju, y para el efecto le apresó al Correjiclor de la Provincia de l'inta !lamato Antonio Ariaga, el dia enatro de Noviembre del año de 7780 , y le ellcerró en un ealaboso cargato de prisiones, y causandole por traidor a la Nacion (7), por los muchos repartos con rue oprimtio a los Fispañoles Americanos, y á los incligenas, pues habia hecho su reparto

(3) Todo el doeumento reposa sobre la errunca tesis de que la robedion del cacigue Tapac Amaru bascabat la independeneia del Perú. los cierto is que perseguia uma ralieal noriljxación itlministrativa, el cetricto cumflimiento de las leyes cladas para las Inctias, ln supresión de abusos lagrlizatdos, por ejemplo, los Repartinientos mercintiles ete. Esto lo pouletuos ol servar por la lectura sle ciertos docnmentos escritos por e] frojio eacidum Túpac Anaru a diversos inclividuos. Véase la "Coleceicin le obran y ctoenmentos relntivos a la historia antigua $y$ moderna de las provineias ilel líu de Ia Plata, pab. Fedro Angelis.-1.2 edición, t. V. Bnonos Aires 1836.

(4) El Curato y pueblo principel del cacicaygo cra Pampatulara, y sus

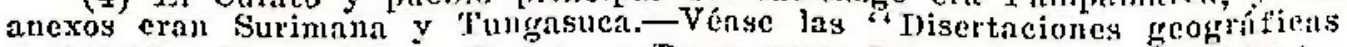
$\checkmark$ cientifiens", por Cosnie Bueno, on Doenmentos Literarios, pub. ML. Otriozola, pp. 93-95.

(5) Vease la "Genenlogia re "T'upae Amaru", pub. Trancisco A. Loaysa, serie $\mathrm{I}$, tomo $\mathrm{X}$. Imp. Miranda. Lima 1946.

(6) Gareía de Loyola iba eomo Capitän, siendo el jefe de la expedieión Martín Furtaclo de Arbieto. Tambioun tomb parte en este suecso el cronistis Perlo Sarmiento de Gamboa, como Alterez real y Secretario.

(7) Lo acusaba de trnihor al Rey, por inoluservalte de sus jeyes parab las rndias. In el fondo esta acusación era cierta, pues los Corregirores cx. torsionabar a los indigenas y burlaban el pago de impuestes a ja Real ha. cienda. 
oi valou de mas 30000 pesos en lugar de 11200 pesos que designaba la tarifal de 'linta por un quinquenio. Hizo llamamiento á todos los Eepajoles Arnerienos é indigenas de dichil Provineia, $y$ en presencia de ellos, en la plaza publiea lo ahoreó el dia 10 de Noviembre del vismo a ịo de 1780 , pisato este acto publico, declaró el levantaniento, y la intencion que tenia de reasumir el Imperio usurpado (8). $A$ lit primera notieial, el Corregidor inmediato de la Provineia de Quispicanchi, que lo era Don Fernando Cabrera y que recidia en Quigitijana, pasí con la mayor asselereion a la Ciudad del Cuzco, donde dio noticia del levantamiento, $y$ la desgracia de Antonio Ariaga (9) ; con esta nueba el correxidor dol Cuzco Don Fernando Valles y Inclan (10), pensó en la defensi, $y$ al efecto echo vando, haciendo llamaniente a toda la gente, para que se alistasen, vajo de las Banderas del Rey. El Señor Obispo Doetor Don Manuel Moscoso, y Peralta (11), dió las Ordenes correspondientes a su Clero, para la pacificacion como Principe de paz, $y$ lenidad.

"Mas el Govierno Seeular, tomo lia medida de crear un Cnerpo conpulesto de siete voeales, $\mathbf{y}$ le priso el nombre de Junta de Guerra: los inclividnos que la componian, fueron los siguientes. Presidente Jon Fernando Valdes y Inclan carallero del Orden de Santiago, Justicia Mayor, lugar Teniente de Capitan General. Don Grabiel ce Abiles (12), que con el timpo vino á ser Virrey del Peru (13). Don Manuel de Villalta Corregidor de Abancay, que con el tiempo vino á ser Brigadier. Don Juan Manuel Canpero. Don Mignel Torrejon Coronel antigno. Don Sebastian Jose Ocampo. Don Mar$\cos$ Antonio de la Camara y Esendero. Don Jonquin Valcarcel Corregidor de la Provincia de Quispicanchi to To fué despues: Ante el Escrivano Tomas de Gamarra.

(s) Vúge la nots N.9 3 .

(9) Vótse la "Representación" de Miguel de Arringa y Eugenio Balra de Berganza ell "La verdid desuuda" pub Franeiseo A. Loayza, serie I, tomo IfI. Imp. Miranda. Lima, 1943 .

(10) Era don Fernando Inelán Valdez.

(11) Vése el Epistolario de Moscoso en "Estaclo del Perú", por José Sahuraura. Pub. Frauciseo A. Lonyza, serie I, tomo V, Pp. 123-221, Inp. Miranta. Isima, 1944.

(12) El entonces coronel Avilés salió de Lima, eon un pequeño cueṛ̣o de socorro formado por 200 hombres del Regimiento de "pardos", el 28 de inaviembre de 1780 . La tropa llevaba fusiles $y$ otros implementos, además de su equipo normal, para los defensores del Cusco. Avilés fué nombralo a última hora, pues don Demetrio Egán, designado primitivamente, habia enfermado. Avilés entró en la ciudad del Cusco el primero de enero de 178T.

(13) Antes había ocupado Avilés el mismo cargo en el virreynato de Buenos Aires. 
"El dia señalado salicron del Cuxeo (14), casi la mayor pielte de sus Vecinos llebaldo tods los articulos de giterrit, pensindo trahar (sic) la Cabesa de liupace Amaro en nna piesa; ufaness eon estoss pensamientos llegaron al pueblo de Sangarara, y como soldados visonios (15) se alojaron en lat Ielecia. Sabida la notiotia por 'lupate: Amaro reunio toda su gente, $y$ a la media noche, serró La Igrlecia. y dando su gente un grito Horrible ateró a todos ellos: a esta hora empesio el insendio de la Iorlecia, los visonios no pudieron escatfar, porque la puerta estaba cots doble guadia, para degollas ib cuantos querian salir, asi es que los que iutentaron por la puerta principal jerecieron, $y$ los que ruedaron corrieron la misma suette aguados todos. del humo del fuego, $y$ de las vigas incondiadas que? caian, de lo alto del templo. La victoria fué tan completa (16), que to dubo guien llebase la noticia al Cuzo. Con la terrible victoria de Santrarará, Tupae Amaro quedó muy satisfocho y los realjstas en el Cuzco se llenaron de miedo y de espanto; pero pasado $r$ ptimer golpe de la imprecion de la imarinacion, sus vecinos se dividieron en vaudos, unos pocos pusilanimes estaban por el Rey. pero los mas por el Principe 'Tupae $\Lambda$ maro (que lo intitulaban yá asi) que como la aurora tlel dia, se asomaba al dar libertad, y Patria, a. lis Nación oprimicla (17).

"La noticia se diviló por torlas partes del Imperio Peruano, la revolucion tomó gran incremento, el sagrado nombre de Inca, é independencia de Fipaña, inflamó los corazones asi de Españoles Americanos, como de los indigenas. Fl bosteso llegó í todas las Gudades, y Provincias, y qaso mas altá de La Paz, Potocí, y Cochatbamba, y en todas chas no se pensaba sino en la libertad de la. Tatria.

"En este cstado, sale del pueblo de Urubamba, un joben gaJlardo de edad de 18 años, animado de la sangre, $y$ de la vengansa, contri los opresoles de sil tierra, y de sus derechos. Este es el bravo Don Andres Tupae Amaro por otro nombre Mendiguri (18), por haber sido hijo lergitimo de un $N$. Mendiguri Español Viscaino, y de la Nusta Doña Felipa Amaro, hermana legitima de Don Jose Gra-

(14) Las tropas eusqueñas estaban bajo cl comando del corregidor do Quispienchis don Fernando Cabrera.

(15) Leasc, bisoños.

(16) La victurja ocurrio el dín 18 re noviembre re 1780. Wl cura Mol]inedo, capellán de los vencislos, la dejado uma relación inédita rel combate.

(17) Véase la nota N.o 3.

(18) Andrés Mencligure tiene figuraeión activa solamente despuess de la captura del cacique José Gabriel Túpac Amaru. El y otroa prominentes rebeldes lograron fugar a la provincia de Aźnguro. 
bicl 'Tupac Amaro (19). Su Tio le hizo General $y$ le confíó una divicion de doce mil hombres, con esta gente, $y$ con los fuegos de un jeben de tan temprana edad, puso espanto y terror en las Provinrias de Huamani, Laricajn, y otras, de este distrito. Puso sitio a la liudad de Sorata (20), a donde se habian refugiado con sus familins, y riquesas los Españoles de las inmediaciones. Sin armas de fuego, no podian los Indios atacar a la Ciudad que estaba fortificasa, pues aungue las obras eran de tierrn, se hallaban defendidas por artilleria; pero el Bravo Don Andres Tupac Amaro venció esta diricultad, empleado un medio que haria honor a cualquier General experlito. Por medio de la construceion de un dique ó espesie de pantano, rennió las aguas que can de las montanias de Ancona cuLiertas de nieve, $y$ dirigiendolas despues de acumuladas en gran canticlad, hacia las obras de tierra que defendian la Cindad inutiles; su impulso destruyó las malas construidas fortificaciones, y su consequencia inmediata fué la toma de ln Ciudad á viva fuerza (21), $y$ el deguello de sus habitantes.

"Pasados algunos meses, los Españoles alcansaron al fin loque sus cerueldades no habian podito lograr. Los dos principales Gefes is decir Don Andres 'Tupac Amaro, y Don Diego Christoval Tupac Amaro (22), en consequencia de haberles pregonado of reciendo artificiosimente sumas conciderables alque los entregara, fueron ven- lidos por sus mismos criados, $y$ por tal medio el Reyno de Ia tirania se prolongo algunos años mas ayudado por la crueldad, y el traude sus compañeros inseparables. Sin embargo esta revolucion. produjo la abolicion del Reparto $(23)$ e en todo 10 demas los Indios han continuado tan oprimidos como antes.

(19) Saluaraura yerra en este pasaje. En realidad Andrés Mendigure, era lijo de Pedro Mendligure, uno de los principnles capitanes del jefe rebe:de Thipac Anaru, y de doñ Ceciiín Típac Amaru, primahermana del Caladillo $y$ hermara de Diego Cristóbai, quien a la maerte del jefe principal da la rebelión continuó dirigiendo la ingurrección, hasta fines de 1781.

(20) El dia 4 de mayo de 1781 .

(21) El dí 5 de agosto de $178 \overline{1}$.

(22) Diego Cristóbal Túpac Amaru firmó un tratado de pax en Lamlea el 11 de noviembre de liss1 con dion Ramón Arias. Este acuerdo fué ratiftcalo en el pueblo de Sichani, al 27 de enero de $17 \bar{z}$, en presencia del mariscal de campo don José del Vniîe y Torres, del obispo Mroscoso y otros altos funcionarios $y$ oficiales que tomaron parte en la segunda expedición punitiva de los españoles contra log rebeldes de las provineias meridionales. Sin cmbargo de tal pacto oficial, el año siguiente (1783) Diego Cristóbal era apresado, so pretexto de estar complicado con los nlborotos ocurridos en el pueblo de Mrareapata e inicindos con la complicidad de su sobrino Ma. riano Túpac Amaru, segundo hij.jo del difunto jefe rebelile José Gabriel.

(23) $O$, tambiél, "f repartimiento mercantii". 
"En este tiempo (2f) Don Jose Ciribiel Tupae Amaro, se asercó a la Cindad del Cuzeo, eon un Exercito formidable, y partio la primera divicion en la accion de Saylla, tres leguas distante de esta ciadad, por un asalto que lijejeron los Españoles í media noche. Jos de Tupae Amaro poco versarlos en la guerra, y sus ardides se vieron sorprehendidos $y$ hubo muela mortandad de parte de los Judios.

"Sabida esta perdida por Tupac Amaro, tomo los altos, $y$ se aparecio en los serros de Puquin de esta Ciudad, los vecinos del Ciuzco se pusieron a la defensa pero de parte de los Chapetones mueho miedo, y pusilanimidad, pues muclos de estos desampararon la Ciudad, y fugaron a la parte de Lima, $y$ los que quedaron llenos de terror, y espanto no supieron dar las Ordenes conbenientes, ni se animaron a ponerse al frente de las tropas del Inca, no obstante que en la Ciudad habia el regimiento de los mulatos de Lima. Alerunos Chapetones llebarlos del miedo se escondieron en las bohedas de las Iglectas. Solo los paisanos eriollos se pusieron en una defensa lenta.

"Salipron de la Cindad dos compañias de los mulatos, al punto de Puquin, los Indios formaron sus enboseadas, $y$ cuando menos pensaron los mulatos les cayeron al cucllo, y fueron muertos a garrotasos torlos ellos, sin que ninguno quedase con vida. El que escribe vio meter al Convento de los Padres Beledmitas (25), como borregos cargados en mulas y cavallitos.

"Al otro dia se aparecio Tupac $A$ maro con su Exercito, y coronó el gran serro de Piecho. No obstante que habian soldados de Linea de los milatós de Lima, ino salieron a la defensa; en este estado lubiera ese dia tomado el Cuzeo Tupac Amaro, sino le hace la traicion su artillero en quien tenia su confianza, llamado Jose Ramon Figueroa Chapeton Gallego, á quien por su traicion le dieron lo: Españoles el sueldo de Coronel de Exercito efectivo en nombre del Rey, y despues le prometieron el grado de Brigadier.

"Por esta traición, y porque Don Mateo Pomacahua $\{26\rangle$ so apareció con su gente armada por la parte de Sacsahuaman eontra su Principe Tupac Amaro, perdió las esperansas, y se retiro como derrotado (27). Estos fueron los principios para que Tupac Ama-

(24) En los primeros dias del mes de enero de 1781.

(25) Léase "bethlemitas".

(26) El cacique Pumacaliua fué un acerbisimo enemigo de la rebelión. En los doeumentos de la époea se encuentran muchas alabanzas de las alltoridadeg por el gue califiearán de "heroico" Cacique do Chineheros.

(27) Este sucedio al amaneeer del 10 de enero de 1781 , día en que se descubrió el inopinado retroceso de los sitiadores. 
ro cayese prisionero en Langui el dia 6 de Abril del año 1781, el referir por menor sus sircunstancias no permite un apunte ligero.

"Cayó en la red el Bravo Tupae Amaro, yá por la traicion de los que le siguieron, yí por los engaños de los chapetones. Fué conducido á esta Ciudad del Cuzco 'inpac Amaro, el que escrive presencio su dentrada, siendo esta pubtica : las ealles por donde havia de pasar estaban con soldudos armados, las ventanas y balcones todas llenas de la gente curiosa, que habian llevados de la novedad. El gue escrive se halló en una de las ventanas de Don Bernardo Rocea my tierno de edad de siete años (28).

"Don Josc Gabriel Tupac Amaro venia sentado como muger (29), en un sillon, con grillos á los pies, la cabesa descubierta para que todos to vieran, trahia un Unco de terciopelo negro con sobre puesto de Oro, en el pecho tenia pendiente por una cadena, una Cruz de Oro, con si Santo christo, las medias de seda blancas, y el sapato de terciopelo negro, el semblante sereno, y la color propia de Incat.

"Tras del desgraciado Inca, venia su muger Doña Micayla Bastidas (30), en una mula, sentada en lun sillón, sin sombrero, para que la conosean.

"Aras venii la Ñusta Doña Tomasa Titu Condemayta casica de sangre de siete pueblos, entre ellos el de Sangarara, donde fué la primera accion de los visoĩos Tsspañoles. La dicha Nusta Doña Tomasa, fué prima hermana de mi Abuela por parte de Madre, la cual se llamaba Dofna Melchora Faurae de Arisa Titu Condemaita hija legitima de Don Benito Yaurae de Arisa, y de Doña Josefa Titu Condemayta. La dicha Doñai Josefa nació emo Sangarará, de donde fué llebadia al pueblo de Oropesa, donde se easó con Don Bewito Yaurae de Arisa, de euyo enlase nació la mencionada doña Melchora: en esta Ccova tubo Don Benito a la Nusta Doria Sebastiana Yaurac de Arisa Titu Condemayta mi Madre, unica hija heredera de los derechos de sangre, y Casicasgos de Muinaparte y Cuzcoparte del pueblo de Oropesa Provineia de Quispicanchi. A mi Abuela Doña Melchora comunmente la titulaban en su pueblo y casicasgo de Oropesa Haton Ceoya, y a mi madre Huchui Ccoya tanto por su

(28) Tíflac Amaru y los principales comprontidos entroban en la cil rad del Cusco el día 14 de abril de $178 \mathrm{t}$, sábado le gloria.

(29) Dada la corta edad dẹ Sahuaraura (7 años), su descripeión parece estar mezclada con lo que oý decir a otros testigos presenciales y to que ocasionalmente pudo habej léilo acerca del moviniento.

(30) Con respecto a las prinejpales mujeres de la rebelión, vénge "Mártires y beroinns", pub. Prancisco A. Losyza, serie I, tomo $\mathrm{XX}$, Tmp. Mirnnสa. Isimn, 1945 . 
tierna edad, cuanto por que la señora grande mi Abuela aun estaha viva.

"Mas el hijo primogenito de Don Jose Grabriel Tupac Amaro liamado Don I frolito Tupac Amaro de edad veinte $y$ un años.

"Mas un Don $X$. Ponse houbre blanco cono de sesenta años. Casicque que fué en el pueblo de Livitaca de Casta blanca.

"Mas un Don Franciseo Tupae Amaro, tío de Don Jose Grabriel Tupac Amaro, hombre al pareçr de sesenta años.

"Mas un Don $N$. Condori Indio principal del pueblo de Charisaca, casique de un ayllo.

"Asi dentraron los desgraciados primogenitos de la Patria, a la Corte Imperial de sus padres eargados de priciones, por haber proyectado dar libertad a su tierra natal oprimida por los tiranos. Fueron alojados en el Convento de los Expatriados Padres de la compañia de Jesirs en calabosos separados. El sruel Vicitador Don José Antonio de Areche les formú la causa $(31)$, y queriendo seharse mas en la sangre de los llustres Peruanos, se atrevio fi visitarle is Don .Jose Grabriel Tupar: Amaru: Dentró al lupar de su pricion eon un pomposo acompañamiento, de Oficiales y Escrivanos, $y$ queriendo aturdir $y$ humillar de un golpe con un aire imperioso le dijo: Digame 'l'upae Amaro, quienes son los complises en esta revelion? al instante sin turbarse, con semblante sereno, $y$ con vos socegada le dijo: Dos son: cuales? respondio Areche Vuestra merced y yo dijo Tupac Amaru: Vuestra merced en poner pechos a la Nacion, $y$ yo en querer quitar, $y$ no hay mas, ni que aberiguar. Respuesta propia de un Principe, pues ni la muerte proxima, ní el estado oprimido, de hallarse con grillos $y$ cadenas pudo poner espanto á ese Corazón magnanimo fno quisocacusar á ninguno, ni que pagasen otios con ruina de su vida y de su familia. Sabia muy bieı ul Ilustre Tupac Amaro quienes eran los compliees, pero ese pecho, on euyas venas, corria la Sangre Real, no quiso por un momento que padeciesen familias, que antes habian sido vasallos de sus Abuelos.

"Areche salió del calaboso muy corrido, y dio orden aqué á Tupac Amaro le pongan en tortura, asta que confiese á los complices de la revelion. El ilustre pricionero, sufrió todo genero de tormentos, cual otro Martir de los primeros siglos de la Era christiana. $\Lambda \mathrm{l}$ fin los que atormentaron á los christianos fueron gentiles, inbuidos en la Doctrina de su paganismo, Doctrina que alagaban los sentidos, y placeres del hombre. Pero chapetones que blasonaban ser

(31) El proceso fué onstanciaclo por el oidor de la Renl Audiencia de Lima don Benito de la Mata Linares. 
christianos exceder en los tormentos de un desgractado christiano st:lo por aberiguar quienes eran, $y$ cebarse en la sangre de sus semejantes?

" En el saion de la pricion de Tupac Amaro, pucieron en la techumbre unos cordeles pendients, con los cuales levantaron su Cuerpo al aire, con los brasos torcidos a las espaldas, y un peso enorme a los pies; se hallaba yá pendiente el desgraciado Tupac Amaro, lleno de dolores por tener todos los miembros dislocados, $y$ en ves de encontrar compacion, era insultado; $y$ apurado con la pregunta, de quienes eran los complices de la revelion: mas el martir de la patria, se afirmaba que solo dos eran: Areche, y el; ó Inca Potriota que supiste dar exemplo a la posteridad en no condenar a tus parientes, a tus amigos, $y$ á tus paisanos, constante en tu exprecion, en defender los derechos de tu pais, afirmaban, que solo Areche era la causa de la revelion, en haber puesto pechos, y el en quitarlos, y dar alivio a los oprimidos. Desde los primeros años en que la Nación dio el sagrado grito de su independencia, y libertad, tubistes millares, que te imitasell: puestos en los patibulos por nuestros opresores, $y$ a la vista de inauditos tormentos, gritaban la libertad de su patria, y la independencia, sin acusar á ninguno de sus semejantes. En diversas partes del nuebo mundo, se han visto heroes con admiración de sus atormentadores, asi en los hombres, como en el vello sexso, de manera que confundidos los chapetones perdieron la esperanza de lieynar en un pais que sus habitantes les Jetestaban de Corazon, y les vomitaban.

"Al fin Tupac Amaro es sentenciado á una muerte de la mas cruel, $y$ que al ver practicarse se horroriso la misma naturaleza. menus el corazon de Areche, $y$ de sus compañeros. T para que se haga cargo la posteridad y que por otra parte no se diga, que yo eomo nacido en el lugar exagero; pongo a la letra la sentencia, $y$ es como sigue". (32).

Dantel VALCÁrcel.

(32) Aqui finnliza el doctmento. La sentencia puede verse en log Doeumentos de Pedro Angelis, citados en la nota N. 3 . Véase asimiamo, en: el tomo I de los Documentos Fistóricos de M. Odriozola. 